\title{
Compressive Current Response Mapping of Photovoltaic Devices Using MEMS Mirror Arrays
}

\author{
Simon R. G. Hall, Matt Cashmore, John Blackburn, George Koutsourakis, and Ralph Gottschalg, Member, IEEE
}

\begin{abstract}
Understanding the performance and aging mechanisms in photovoltaic devices requires a spatial assessment of the device properties. The current dominant technique, electroluminescence, has the disadvantage that it assesses radiative recombination only. A complementary method, laser beaminduced current (LBIC), is too slow for high-throughput measurements. This paper presents the description, design, and proof of concept of a new measurement method to significantly accelerate LBIC measurements. The method allows mapping of the current response map of solar cells and modules at drastically reduced acquisition times. This acceleration is achieved by projecting a number of mathematically derived patterns on the sample by using a digital micromirror device (DMD). The spatially resolved signal is then recovered using compressed sensing techniques. The system has fewer moving parts and is demonstrated to require fewer overall measurements. Compared with conventional LBIC imaging using galvanic mirror arrangements or $x y$ scanners, the use of a DMD allows a significantly faster and more repeatable illumination of the device under test. In this proof-of-concept instrument, sampling patterns are drawn from Walsh-Hadamard matrices, which are one of the many operators that can be used to realize this technique. This has the advantage of the signalto-noise ratio of the measurement being significantly increased and thus allows elimination of the standard lock-in techniques for signal detection, reducing measurement costs, and increasing measurement speed further. This new method has the potential to substantially decrease the time taken for measurement, which demonstrates a dramatic improvement in the utility of LBIC instrumentation.
\end{abstract}

Index Terms-Compressed sensing, digital micromirror device (DMD), laser beam-induced current (LBIC) measurements, photovoltaic (PV).

\section{INTRODUCTION}

$\mathbf{S}$ OLAR cells or photovoltaic (PV) devices are an essential part of the increasing worldwide investment in renewable energy generation. Confidence in the production of viable

Manuscript received September 26, 2015; revised March 23, 2016; accepted March 24, 2016. This work was supported in part by the European Metrology Research Programme within EURAMET and the European Union through the Project ENG55 PhotoClass, in part by the U.K. National Measurement System, in part by the European Commission FP7 through the Project SOPHIA (INFRA-2010-1.1.22_CP-CSA-Infra) under Grant 262533, and in part by the U.K. Research Councils through the Project Stability and Performance of Photovoltaics under Contract EP/H040331/1. The Associate Editor coordinating the review process was Dr. Mark Yeary.

S. R. G. Hall, M. Cashmore, and J. Blackburn are with the National Physical Laboratory, Teddington TW11 0LW, U.K.

G. Koutsourakis and R. Gottschalg are with the Centre for Renewable Energy Systems Technology, Loughborough University, Loughborough LE11 3TU, U.K. (e-mail: simon.hall@npl.co.uk).

Color versions of one or more of the figures in this paper are available online at http://ieeexplore.ieee.org.

Digital Object Identifier 10.1109/TIM.2016.2559878 amounts of electrical energy relies on adequate metrology, both at production level and during use [1], [2]. Previously, electrical measurements have been preferred to optical techniques for power generation monitoring due to the perceived cost and complexity of optical photocurrent instrumentation, even though the ability to characterize existing and developing faults at panel level would enable the PV system electrical energy production to be maximized. Comparisons of alternative PV technologies [3] rely on assumptions of the uniformity of the PV modules. A well-defined photocurrent map would allow an improved uncertainty budget to be established which would allow reasonable comparisons between PV devices. We describe a low-cost high-speed system, which could be used to achieve a photocurrent quantum efficiency map of PV panels for both operational optimization and quality control during manufacture. This would improve the level of control by identifying panels with regions of differing response, which could develop into performance-limiting defects or identification of nonideal uniformity due to contamination or dirtying [1]. In-process monitoring would reveal the evolution of problematic regions at panel level and would allow early action to maintain the PV system energy production. PV devices are in many cases limited by localized flaws in the material and thus it is important to probe the spatial characteristics of solar cells. Common nondestructive methods are electroluminescence (EL) imaging [4], lock-in thermography [5], and photoluminescence [6], which are complementary to each other. Luminescent methods have the advantage of being faster but are limited in what they can probe as they rely, as the term implies, on luminescent recombination processes. This gives valuable information if the dominant recombination mechanism is luminescence. In the case of the prevalent technology (crystalline silicon PV devices), this is questionable as in many cases the dominant process is surface recombination, which is not a luminescent process. Other technologies such as thin-film solar cells are affected by series resistance and render EL measurements difficult. Nevertheless, some flaws such as cracks in wafers can be easily identified, and thus, EL is a common technique but gives very little information on the status of the semiconductor.

A method capable of accurately assessing the entire semiconductor current map is the laser beam-induced current (LBIC) method. In such systems, a laser beam is sequentially scanned across the PV sample, as illustrated in Fig. 1(a), utilizing an $x y$ translation stage for moving the sample [7]; changing the direction of the beam with a piezo-controlled 


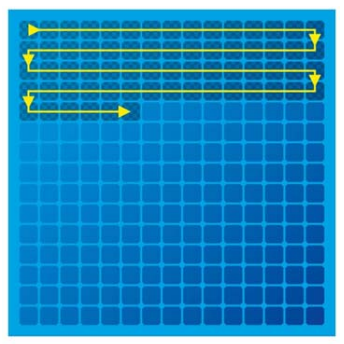

(a)

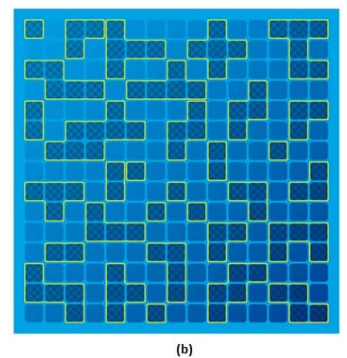

(b)
Fig. 1. (a) With an LBIC standard raster scan, each measurement is taken with illumination of a single point. Successive measurements record the signal as the illuminated spot is scanned systematically over the surface of the PV, recording the current response of each illuminated area. (b) Illumination of multiple points simultaneously for each measurement, so that each measurement covers a much greater surface area of the PV than for standard LBIC using a raster scan.

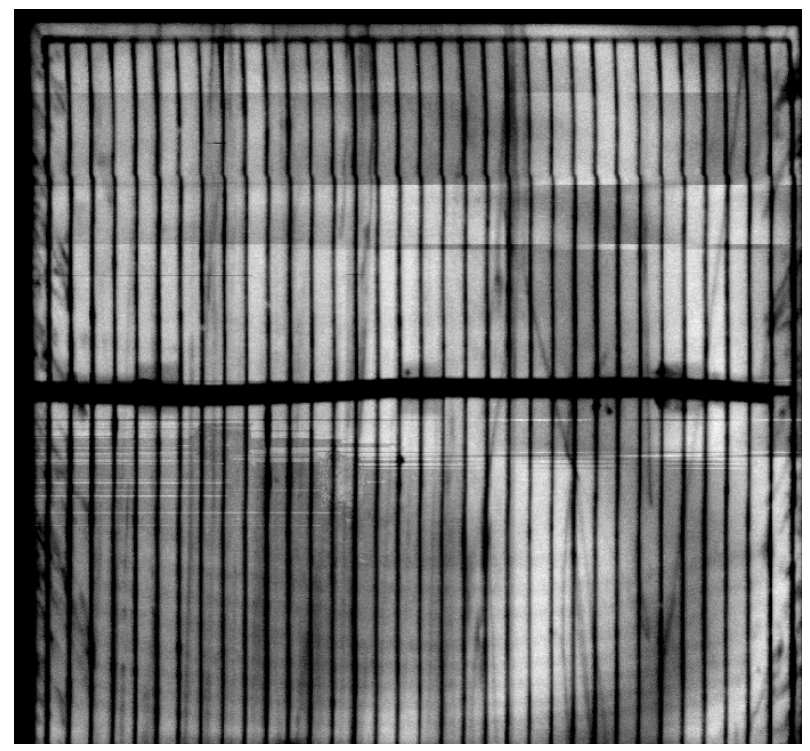

Fig. 2. Example of an LBIC image where thermal instability affected the measurement, as can be seen in the top half of the cell.

mirror [8], [9], or scanning the laser head with movement stages [10]. The photocurrent generated is recorded as a function of position, producing the final map. The irradiance produced by the laser produces a small current and thus generally lock-in detection is required to obtain meaningful signal-to-noise ratios (SNRs).

The standard LBIC method is the only commercially available method that provides reliable current mapping results, but it is generally considered to be too slow for larger samples. Consequently, it is mainly applied to research cells that have a very small area or when measurement time is not an important factor. The extended time it can take to measure a sample can result in measurement artifacts. An example of experimental artifacts altering the photocurrent map is presented in Fig. 2. When current mapping is implemented in a large chamber as in the experimental setup described in [9], air flow failure can lead to temperature instability in the chamber that can result in small displacements of the beam, which are visible in the top half of the current map in Fig. 2.

A further disadvantage of the standard LBIC technique is that it is only applicable to individual PV cells.
LBIC photocurrent mapping of PV modules presents significant problems as described in [11]. These experimental issues are compounded by long measurement runs, which increase the possibility that variations in temperature and environment can generate artifacts in the resultant current map, as shown in Fig. 2. The small photocurrent generated by the raster scanning laser is more susceptible to electrical noise, because the noise will form a larger percentage of the signal than in the compressive sensing (CS) approach.

We demonstrate a radically enhanced LBIC technique using compressed sensing that significantly reduces measurement time. The technique improves data collection, by illumination of approximately half the PV for each measurement; a much larger photocurrent is produced with a consequent noise reduction due to an improved SNR, thus removing the need for lockin detection. To achieve the reduction of measurement time, a digital micromirror device (DMD) [12] is used to selectively illuminate the PV while the compressed sensing sampling theory is used for retrieval of the photocurrent map [13]-[15].

CS uses a priori knowledge that the relevant data in the set of measurement values can have a sparse representation after a transformation. This is based on the fact that almost all natural signals are sparse or compressible, meaning that they are sparse after a suitable transform. This methodology radically reduces the number of measurements required for photocurrent mapping. We describe our implementation of this technique as compressed mapping, in which a series of patterns is projected on the PV cell using the DMD, while the patterns are derived from a frame or basis in which the actual current map of the device is sparse so that the CS theory can be applied. It has been recently demonstrated that a DMD projector can be used to scan a spot of light across the sample instead of a moving laser beam, improving performance in terms of speed over large areas because mechanical translation stages are not required [16]. This approach, in common with standard LBIC, only illuminates a tiny fraction of the PV cell at any one time, generating small currents that require a sensitive current measurement regime. The entire area must be scanned using a focused laser spot to produce the current response map. The method presented here uses patterns with a sparsity of approximately $50 \%$ to illuminate the sample, which is a single PV cell. The use of varying illumination patterns enables the use of compressed sensing algorithms to identify and reconstruct the complete photocurrent map while significantly reducing the number of measurements required [Fig. 1(b)].

\section{EXPERIMENTAL SETUP}

\section{A. System Description}

The prototype system using a DMD and laser illumination for compressive mapping of PV devices is shown in the simplified schematic diagram in Fig. 3. Electrical measurements of the PV cell's current response are achieved using a Vinculum SP042-series current-tovoltage amplifier while the voltage output is measured using a National Instruments NI USB 6211 Analogue to Digital (AD) converter. The fiber-coupled output of a 636.2-nm 1-mW pigtailed diode laser is collimated and directed onto a DMD (Texas Instruments 0.7 XGA DMD 


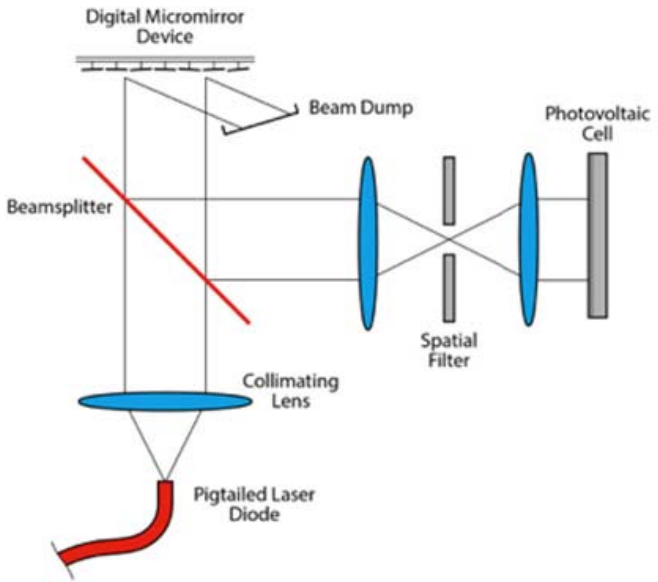

Fig. 3. Schematic of the NPL PV compressed mapping system. This allows a well-defined irradiance distribution to be produced on the PV. The spatial filter removes diffraction artifacts generated by the DMD.

Discovery), programmed to generate the series of patterns required for the measurements. The tilt of the individual mirrors in the micromechanical array can be switched between two distinct angles. This allows light to be redirected either onto a beam dump for unilluminated areas, or onto a 50:50 beam-splitter (for initial irradiance measurement) to produce the illuminated pattern on the PV. Diffraction artifacts are removed using a spatial filter. The PV cell is placed in a conjugate plane in relation to the DMD to ensure well-defined pattern reproduction. One of the advantages resulting from the use of a DMD as a pattern generator is that there is no additional need for moving parts, such as an $x y$ stage or a mirror, that a standard LBIC system would require to scan the PV under test.

\section{B. Compressive Mapping Technique}

The measured signal is the sum of the sparse local current response of the PV device under the structured illumination pattern and successive measurements of these will enable reconstruction of the entire current map. This current response map is a compressible signal, as it can be sparsely represented with a suitable basis set, such as the discrete cosine transform. This provides the ability to apply CS in order to measure the necessary coefficients in fewer steps. The reconstruction of the current map requires the solution of a highly underspecified series of simultaneous equations. Two algorithms common in the field of compressed sensing are used in this paper, $\ell_{1}$ norm [14] and total variation (TV) norm minimization [17]. In essence, the $\ell_{1}$ norm minimization method is suitable for recovery of signals where a large number of coefficients are zero, while the TV norm is optimal for the recovery of signals that have a low degree of spatial variability. The method is demonstrated by characterizing a $1 \mathrm{~cm}^{2}$ dye-sensitized solar cell produced on site. The sample exhibited a high degree of spatial nonuniformity and hence was an ideal candidate to demonstrate the capability of the compressive mapping technique against the conventional LBIC methodology. The test sample was overfilled by the conjugate image of the DMD to ensure complete coverage. The output voltage from the current-to-voltage amplifier was acquired for every projected pattern, and the electrical current-to-voltage amplifier and AD voltage measurement system were calibrated across the input range to ensure measurement system linearity. To suppress dark current contributions from the PV cell, the mean of the recorded current for the binary pattern illumination was subtracted from the current produced by the corresponding inverse pattern. Each recorded voltage measurement from the current-to-voltage amplifier and its corresponding binary Walsh-Hadamard projection matrix were then processed using the TV and $\ell_{1}$ norm algorithms to produce a reconstructed current response map.

\section{Projected Patterns}

The projected patterns that generate the measurements which correspond to the measurement matrices used in compressed mapping are taken from successive lines of the Walsh-Hadamard matrix [18], a binary orthogonal matrix. This can be implemented using a DMD as an alternative to the raster scan of identity matrices, while also retaining their orthogonality condition. A significant advantage associated with using these patterns for data acquisition is that since they illuminate approximately half of the PV sample with each pattern, the SNR is significantly greater than those of similar single-pixel or line illumination methods [19], without the need for a bias light. The Walsh-Hadamard transmission coefficients are 0 and 1 , and were rescaled to +1 and -1 for data analysis to ensure the reconstruction algorithm converged.

\section{Performance Metrics for Compressed Mapping}

To quantify the benefit of the new technique, the reconstructed current response map was compared with the one obtained using a raster-scanned LBIC measurement with the same DMD experimental configuration. The correlation coefficient between CS sampling and raster scan results was calculated to provide the required comparison. As the number of projected Walsh-Hadamard patterns increased, the reconstructed response map increasingly corresponded to the raster-scanned LBIC map (Fig. 4). The value of the correlation coefficient as a function of number of measurements between normalized response maps from the raster scan and CS sampling is presented in Fig. 5.

The formula for Pearson's coefficient $r$ where one dataset $\left\{x_{1}, \ldots, x_{n}\right\}$ is correlated with another dataset $\left\{y_{1}, \ldots, y_{n}\right\}$ both containing $n$ values is given by

$$
r=r_{x y}=\frac{\sum x_{i} y_{i}-n \bar{x} \bar{y}}{\sqrt{\sum x_{i}^{2}-n \bar{x}^{2}} \sqrt{\sum y_{i}^{2}-n \bar{y}^{2}}} .
$$

A perfectly reconstructed map would have a Pearson's linear correlation coefficient of unity, which is equivalent to a 1-to-1 match between all data points. This can be seen in both graphs of Fig. 5, as the number of projected patterns increases (number of measurements), the correlation coefficient increases, regardless of the applied reconstruction algorithm. Despite the improved SNR that results from the larger photocurrent generated by illuminating approximately half of the PV surface at any one time, the noise background 


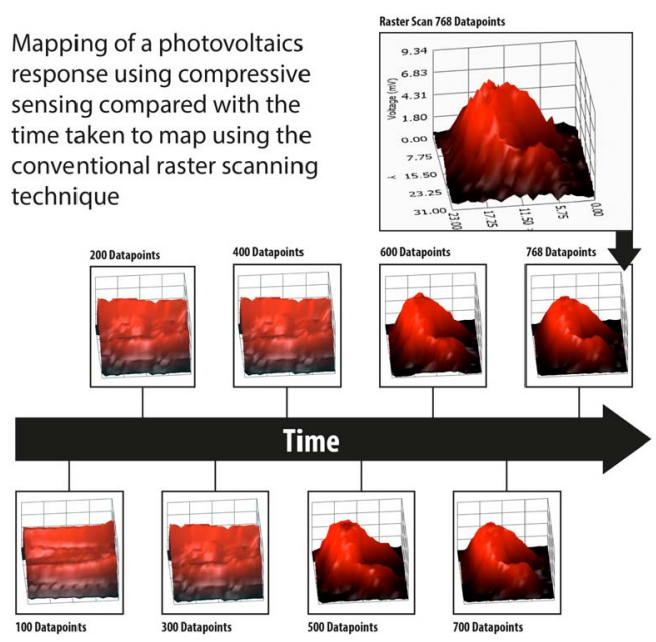

Fig. 4. Comparison of speed between the LBIC technique and compressed mapping using the same mirror array. The time axis is relative, assuming a similar measurement time for each point, and the compressive mapping process will be inherently faster because fewer points are required than for a full raster scan.
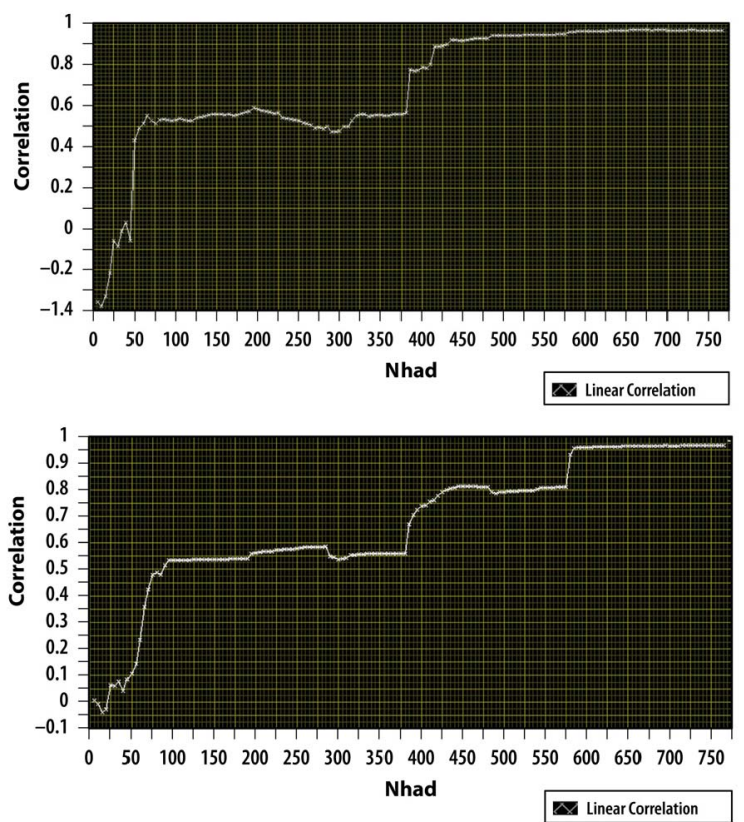

Fig. 5. Comparison of the growth of the correlations between the raster scan of a PV test piece and the recovered map obtained through the compressed sensing algorithm as a function of increasing numbers of Walsh-Hadamard patterns. Bottom graph: this growth for the $\ell 1$ norm minimization algorithm. Top graph: this behavior for the TV technique.

between the two analysis methods is significantly different. Consequently, complete correlation would never occur. This is due to the low irradiance delivered to the sample from the eyesafe laser source, especially in the case of a raster scan, where only one of the pixels of the DMD is in the on state for each measurement. This substantially reduces the optical power delivered to the PV cell causing photocurrent generation to be in a nonlinear regime. These factors preclude a quantitative comparison between LBIC methods, although results are sufficiently clear to provide a qualitative comparison.

\section{PROOF-OF-CONCEPT DEMONSTRATION FOR DYE-SENSITIZED SOLAR CELLS}

It is useful to compare the current response map produced using compressed sensing with an equivalent standard LBIC measurement method, i.e., a complete raster scan with the same setup. Fig. 5 shows that using increasing numbers of Walsh-Hadamard patterns affects the correlation between these two measurements results for both the $\ell 1$ norm and TV norm algorithms. The correlation from the TV algorithm rises and plateaus more rapidly than the plot of the $\ell 1$ norm minimization technique. This is a consequence of the fact that there is a higher degree of sparsity in the variation of our response map than there is in the actual values present. Noteworthy in Fig. 5 are the sudden jumps in correlation in the behavior of both reconstruction techniques. These rapid increases in reconstruction accuracy correspond strongly to the inclusion of specific large magnitude photocurrent measurements noted during data acquisition. This indicates that the signal recorded for specific patterns includes more information than others.

Using the TV norm reconstruction algorithm, we achieve a $90 \%$ correlation to the raster scan current response map when using approximately $55 \%$ of the measurements required for conventional LBIC (see the top graph in Fig. 5). This can be compared to the approximately $70 \%$ correlation when using a similar number of points in the $\ell 1$ norm reconstruction. To achieve more than $95 \%$ accuracy, approximately 600 out of a total of 768 data points were needed. This shows that it is possible that the majority of the current response map information can be recovered in fewer measurements using the compressed-mapping technique than with the raster-scanning method standard LBIC measurement system, using the same DMD. This demonstrates a significant time reduction for photocurrent mapping using the CS technique.

The experimental system provides validation that the concept of compressive sampling is feasible for current mapping of PV cells, as current maps have been acquired with fewer measurements than required for a raster scan. However, the system is not yet optimized in terms of speed. The simple instrumentation used in this prototype experimental setup had a limited sampling rate of $0.5 \mathrm{~s}$ per measurement. In an optimized system, the high SNR of this method can allow very high sampling rates. In addition, the offline response map reconstruction processing implemented in $\mathrm{C}++$ code requires a similar amount of time as the data acquisition. This can be greatly improved by improving the optimization algorithms and measurement quality and using increased processing power or alternative programming platforms (e.g., field-programmable gate arrays).

An improved implementation of the technique will require an improvement of the spatial uniformity of the illumination. The existing experimental configuration uses a Gaussian laser beam spatial profile, which produces an irradiance gradient decrease of $10 \%$ at the edges of the PV from the central maximum. This irradiance variation convolves with the reconstructed signals, and a nonlinear PV cell response introduces an undesirable uncertainty contribution to the experimental 
results. A higher power optical source and a uniform irradiance at the mirror array would improve the measurement by ensuring that the device photocurrent response is measured in the linear portion of its operational range.

\section{CONCLUSION}

In this paper a novel compressed mapping technique is proposed, and is experimentally demonstrated for the first time. This has the potential to be a fast industrial and research measurement method for the acquisition of the local current response of PV devices. High-speed scanning enabled by the technique reduces the generation of measurement artifacts from laser power variation and environmental effects, which can seriously compromise conventional LBIC results. The use of projected orthogonal illumination patterns as an alternative to raster scanning is presented and the reconstruction of a feature map using fewer measurements than would be required for conventional LBIC raster scanning with a high degree of correlation has been highlighted. The development of the technique further by using different transform domains, dictionaries, and reconstruction algorithms will be explored, improving both resolution and measurement speed. Combining the advantages discussed in this paper, a significant reduction of measurement time is achievable, while fewer moving parts in the instrumentation for PV photocurrent mapping results in a less complex, lower cost, higher accuracy system, which demonstrates a clear advance in the instrumentation and measurement of PV cells and modules. The use of high-power optical sources with DMD modulators as used in media projection equipment could allow the measurement of installed PV modules using a similar experimental configuration, revealing lifetime limiting defects and areas of low quantum efficiency.

\section{REFERENCES}

[1] B. Ando, S. Baglio, A. Pistorio, G. M. Tina, and C. Ventura, "Sentinella: Smart monitoring of photovoltaic systems at panel level," IEEE Trans. Instrum. Meas., vol. 64, no. 8, pp. 2188-2199, Aug. 2015.

[2] S. Silvestre, L. Sentis, and L. Castaner, "A fast low-cost solar cell spectral response measurement system with accuracy indicator," IEEE Trans. Instrum. Meas., vol. 48, no. 5, pp. 944-948, Oct. 1999.

[3] A. Carullo and A. Vallan, "Outdoor experimental laboratory for long-term estimation of photovoltaic-plant performance," IEEE Trans. Instrum. Meas., vol. 61, no. 5, pp. 1307-1314, May 2012.

[4] T. Fuyuki, H. Kondo, T. Yamazaki, Y. Takahashi, and Y. Uraoka, "Photographic surveying of minority carrier diffusion length in polycrystalline silicon solar cells by electroluminescence," Appl. Phys. Lett., vol. 86, no. 26, p. 262108, 2005.

[5] O. Breitenstein, J. P. Rakotoniaina, and M. H. Al Rifai, "Quantitative evaluation of shunts in solar cells by lock-in thermography," Prog. Photovolt. Res. Appl., vol. 11, no. 8, pp. 515-526, Dec. 2003.

[6] K. Bothe, G. H. Bauer, and T. Unold, "Spatially resolved photoluminescence measurements on $\mathrm{Cu}(\mathrm{In}, \mathrm{Ga}) \mathrm{Se}_{2}$ thin films," Thin Solid Films, vols. 403-404, pp. 453-456, Feb. 2002.

[7] J. Martín, C. Fernández-Lorenzo, J. A. Poce-Fatou, and R. Alcántara, "A versatile computer-controlled high-resolution LBIC system," Prog. Photovolt. Res. Appl., vol. 12, no. 4, pp. 283-295, Jun. 2004.

[8] J. Carstensen, G. Popkirov, J. Bahr, and H. Föll, "CELLO: An advanced LBIC measurement technique for solar cell local characterization," Solar Energy Mater. Solar Cells, vol. 76, no. 4, pp. 599-611, Apr. 2003.

[9] P. Vorasayan, T. R. Betts, A. N. Tiwari, and R. Gottschalg, "Multi-laser LBIC system for thin film PV module characterisation," Solar Energy Mater. Solar Cells, vol. 93, nos. 6-7, pp. 917-921, Jun. 2009.

[10] H. Cossutta, K. Taretto, and M. Troviano, "Low-cost system for micrometer-resolution solar cell characterization by light beam-induced current mapping," Meas. Sci. Technol., vol. 25, no. 10, p. 105801, Aug. 2014.

[11] P. Vorasayan, T. R. Betts, and R. Gottschalg, "Limited laser beam induced current measurements: A tool for analysing integrated photovoltaic modules," Meas. Sci. Technol., vol. 22, no. 8, p. 085702, Aug. 2011.

[12] L. J. Hornbeck, "The DMD projection display chip: A MEMS-based technology," MRS Bull., vol. 26, no. 4, pp. 325-327, 2001.

[13] D. L. Donoho, "Compressed sensing," IEEE Trans. Inf. Theory, vol. 52, no. 4, pp. 1289-1306, Apr. 2006.

[14] J. Ma, "Compressed sensing for surface characterization and metrology," IEEE Trans. Instrum. Meas, vol. 59, no. 6, pp. 1600-1615, Jun. 2010.

[15] E. J. Candés, J. K. Romberg, and T. Tao, "Stable signal recovery from incomplete and inaccurate measurements," Commun. Pure Appl. Math., vol. 59, no. 8, pp. 1207-1223, 2006.

[16] J.-K. Yoo, S. K. Kim, D.-H. Lee, and S.-N. Park, "Spatial uniformity inspection apparatus for solar cells using a projection display," Appl. Opt., vol. 51, no. 20, pp. 4563-4568, 2012.

[17] E. J. Candès, J. Romberg, and T. Tao, "Robust uncertainty principles: Exact signal reconstruction from highly incomplete frequency information," IEEE Trans. Inf. Theory, vol. 52, no. 2, pp. 489-509, Feb. 2006.

[18] L. Gan, T. T. Do, and T. D. Tran, "Fast compressive imaging using scrambled block Hadamard ensemble," in Proc. Eur. Signal Process. Conf., Aug. 2008, pp. 1-5.

[19] R. Gupta and O. Breitenstein, "Digital micromirror device application for inline characterization of solar cells by tomographic light beaminduced current imaging," Proc. SPIE, vol. 6616, p. 661600, Jun. 2007.

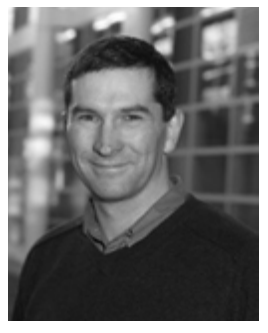

ISO committees.
Simon R. G. Hall is currently a Senior Research Scientist with the National Physical Laboratory (NPL), Teddington, U.K. He is the Lead Scientist for Adaptive Optics with NPL and participates in the European metrology research projects PhotoClass and ThinErgy. He is a Visiting Professor with the School of Computing and Engineering, University of Huddersfield, Huddersfield, U.K.

Mr. Hall is the Chairman of the British Standards Optics and Photonics Committee, and a member and the National Expert for the CIE, IEC, and

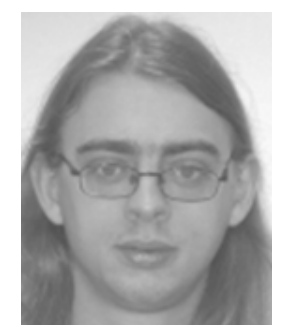

Matt Cashmore received the master's degree in theoretical astronomy and the Ph.D. degree in holographic interferometry from Durham University.

He spent eight years with Durham University, Durham, U.K. He is currently a Research Scientist with the National Physical Laboratory, Teddington, U.K., where he is developing novel optical instrumentation methods for surface analysis and photovoltaic applications.

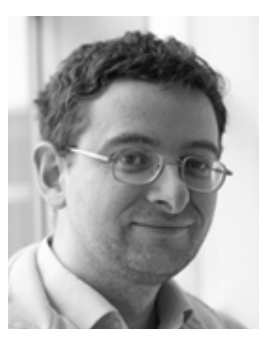

John Blackburn is currently a Senior Research Scientist with the Mathematics and Modelling Group, National Physical Laboratory, Teddington, U.K. $\mathrm{He}$ has been involved in a variety of projects over 16 years, including antenna/resonator design, piezoelectric modeling, fuel cells, molecular modeling, and compressed sensing. He is the author of ZINC, a free multiphysics finite element software package. His current research interests include solid state physics, electromagnetics, and image analysis. 


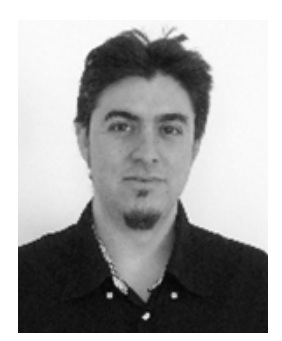

George Koutsourakis was born in Athens, Greece, in 1984. He received the Degree in applied mathematics and physical science from the National Technical University of Athens, Athens, participating in research projects for atmospheric monitoring for application in cosmic ray detection, and the M.Sc. degree in energy and environment from the University of Patras, Patras, Greece, focusing on outdoor performance of thin film solar cells. $\mathrm{He}$ is currently pursuing the Ph.D. degree with the Centre of Renewable Energy Systems Technology, Loughborough University, Loughborough, U.K.

His current research interests include spatial characterization of photovoltaic devices using compressed sensing techniques.

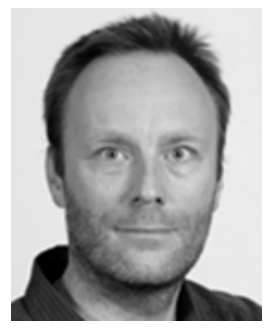

Ralph Gottschalg (M'12) was born in Karlsruhe, Germany. He received the Diploma degree in physics from the University of Karlsruhe, Karlsruhe, and the M.Sc. degree in renewable energy systems technology and the Ph.D. degree in photovoltaic device field performance variation from the Centre for Renewable Energy Systems Technology, Loughborough University, Loughborough, U.K.

$\mathrm{He}$ is currently a Professor of Applied Photovoltaics. 